\title{
Food for Thought: Have We Been Giving the Wrong Dietary Advice?
}

\author{
Zoë Harcombe $^{1}$, Julien S. Baker ${ }^{1}$, Bruce Davies ${ }^{2}$ \\ ${ }^{1}$ Institute of Clinical Exercise and Health Science, Faculty of Science and Technology, University of the West of Scotland, Hamilton, \\ UK; ${ }^{2}$ University of Glamorgan, Pontypridd, UK. \\ Email: zoe@theobesityepidemic.org
}

Received January $16^{\text {th }}$, 2013; revised February $18^{\text {th }}$, 2013; accepted February $25^{\text {th }}, 2013$

\begin{abstract}
Background: Since 1984 UK citizens have been advised to reduce total dietary fat intake to $30 \%$ of total energy and saturated fat intake to 10\%. The National Institute of Clinical Excellence [NICE] suggests a further benefit for Coronary Heart Disease [CHD] prevention by reducing saturated fat [SFA] intake to $6 \%-7 \%$ of total energy and that 30,000 lives could be saved by replacing SFAs with Polyunsaturated fats [PUFAs]. Methods: 20 volumes of the Seven Countries Study, the seminal work behind the 1984 nutritional guidelines, were assessed. The evidence upon which the NICE guidance was based was reviewed. Nutritional facts about fat and the UK intake of fat are presented and the impact of macronutrient confusion on public health dietary advice is discussed. Findings: The Seven Countries study classified processed foods, primarily carbohydrates, as saturated fats. The UK government and NICE do the same, listing biscuits, cakes, pastries and savoury snacks as saturated fats. Processed foods should be the target of public health advice but not natural fats, in which the UK diet is deficient. With reference to the macro and micro nutrient composition of meat, fish, eggs, and dairy foods the article demonstrates that dietary trials cannot change one type of fat for another in a controlled study. Interpretation: The evidence suggests that processed food is strongly associated with the increase in obesity, diabetes, CHD, and other modern illness in our society. The macro and micro nutrients found in meat, fish, eggs and dairy products, are vital for human health and consumption of these nutritious foods should be encouraged.
\end{abstract}

Keywords: Cardiovascular Disease; Obesity; Fatty Acids; Saturated Fatty Acids; Monounsaturated Fatty Acids; Polyunsaturated Fatty Acids; Dietary Fats; Dietary Carbohydrates; Epidemiology

\section{Introduction}

History has presented us with a very confused and sometimes misinformed message with reference to healthy eating. Following the completion of the Seven Countries Study in 1970, the seminal work of Ancel Keys [1] had a profound influence on the diets of the USA [2] and the UK [3]. Keys' conclusions were:

1) The incidence rate of CHD [Coronary Heart Disease] tends to be directly related to the distributions of serum cholesterol values.

2) The average serum cholesterol values of the cohorts tended to be directly related to the average proportion of calories provided by saturated fats in the diet.

3) The CHD incidence rates of the cohorts are as closely related to the dietary saturated fatty acids as to the serum cholesterol level.

\section{Dietary Advice}

In 1983 these findings prompted the National Advisory Committee on Nutrition Education [NACNE], to rec- ommend a reduction in total dietary fat intake to 30\% with saturated fatty acids being no greater than $10 \%$. In 1984 this was followed by the Committee on Medical Aspects of Food Policy [COMA] policy paper "Diet and Cardiovascular Disease [4]." This report endorsed the findings of Keys stating that the dietary energy derived from saturated fatty acids tends to be related to mortality from CHD and that this relationship is consistent between countries.

The 2010 public guidance document from the National Institute of Clinical Excellence [NICE] [5] entitled "Prevention of Cardiovascular Disease at a Population Level" also emphasised that the reduction of dietary saturated fat is crucial to the prevention of CHD. They recommended that a halving of the average intake (from $14 \%$ to $7 \%$ ) might prevent 30,000 deaths annually. Personal correspondence with NICE with reference to these recommendations confirms that they were very much influenced by the work of Harris et al., 2009 [6]. In retrospect it would seem that the work of two research groups i.e. Keys et al., 1970 [1] and Harris et al., 2009 [6] have con- 
siderably influenced the conclusions and recommendations of two of the most important and influential health reports that have been published in the last quarter of a century.

When we examine the work of these two research groups it is surprising how their results and conclusions were accepted with such conviction and more importantly superimposed on society to such an extent that they determine UK eating habits.

\section{Classification Errors}

Volume XVII of Keys' study is called "The Diet" [7] and yet there is very little mentioned about the diet of any of the countries studied. Each country is discussed in a separate volume and there is no scientific quantification of the foods consumed by any of the countries involved in the study. The dietary references that were mentioned used unquantifiable descriptions such as "loaded with saturated fatty acids" and "cholesterol from butter, cream, meats and eggs".

More importantly the study classified cake and ice cream as saturated fats, as opposed to refined carbohydrates, an error which is repeated by contemporary food scientists. Meat and eggs are described as saturated fat when their fat content is primarily unsaturated. Butter and cream are one third unsaturated fat, which was not noted in their analysis. So here we have a profoundly influential research project introducing imprecise evaluations of macronutrients which have continued to the present day.

The Seven Countries Study was not a scientifically robust study. The dietary references are vague, sporadic or absent. There were no comments on causation and no attempt was made to consider association until 25 years post study completion [8] and 10 - 15 years after UK and USA dietary advice had already been changed based on the recommendations of Keys' work. The study clearly demonstrated that the science surrounding macronutrients and nutrition was not as accurate as it is today. Yet contemporary knowledge is not being applied when considering nutritional advice for the population.

\section{Saturated Fats and Polyunsaturated Fats}

Harris and his colleagues [6], using a meta analysis review, were of the opinion that consumption of at least $5 \%$ to $10 \%$ energy from omega- 6 polyunsaturated fats [PUFAs] reduces the risk of CHD relative to lower intakes. They were confident about the safety of higher intakes of these fats. The conclusions to date are equivocal with reports from robust research concluding that diets high in PUFAs can increase the susceptibility to LDL [low density lipoprotein] oxidation and vascular inflammation [9].
In addition, Mozaffarian et al. [10], using angiography, reported a direct association between PUFA intakes and luminal narrowing in women with CHD. To date there is no population study that has consumed large quantities of polyunsaturated fatty acids for extended periods of time. Therefore the benefits from the consumption of high intakes of PUFAs have not been proven to be safe [11]. Because of the important impact that PUFAs are claimed to have on cardiovascular health it is critical that research disclosures are appraised with care to identify any conflict of interest with the sponsors of such projects.

Harris et al. excluded two studies, Rose [12] and Frantz [13], which were unfavourable claiming no significant effect was seen. Selecting six studies [14-19] for their analysis as opposed to eight studies does present a confounding variable when considering their conclusions.

\section{Nutrient Classification}

It would seem that two influential nutritional recommendations written during the period from 1980-2010 were the COMA [3] and NICE [5] reports and that the writing of these reports was in turn considerably influenced by the research of Keys [1] and Harris [6]. To date we have been guilty of imprecision in the identification and classification of foods. This leads to dietary recommendations, based on incorrect information and in many instances poor science.

There is a need to accurately define the macro and micronutrient content of food. Biscuits, savoury snacks and processed food should not be defined as saturated fats because they are substantially carbohydrates. Red meat is not a saturated fat but a combination of various fatty acids. Sirloin steak for example is approximately $71 \%$ water, $21 \%$ protein, $3 \%$ unsaturated fat and $2 \%$ saturated fat $[20,21]$. Natural food such as meat, fish, eggs and nuts contain saturated, monounsaturated and polyunsaturated fats, only the proportions vary. Few people appreciate that it is impossible to eat saturated or polyunsaturated fat alone. Dairy products are the only food group with more saturated than unsaturated fat. Many of the foods demonised by past research groups, even lard, contain more unsaturated than saturated fat. Dietary fat consumption is a key provider of essential fats and fat soluble vitamins.

The only foods that contain no carbohydrate or protein are oils $[22,23]$. Sucrose contains only carbohydrate, no fat or protein. Every other food contains protein with fat, carbohydrate or both. The only food that can be modified to assess the dietary implications of fat consumption without changing the macronutrient composition is oil. This explains why the COMA [3] report stated "There has been no controlled clinical trial of the effect of decreasing dietary intake of saturated fatty acids on the 
incidence of coronary heart disease nor is it likely that such a trial will be undertaken”.

\section{Dietary Fat and Coronary Heart Disease}

The Heart of Mersey Paper [THoMP] [24], which considered dietary fats and the prevention of coronary heart disease, concluded that saturated fats are a clear agonist for the development of CHD with the unsaturated fats assuming an antagonist and beneficial influence. Using a $100 \mathrm{~g}$ steak, as an example, with $5.4 \mathrm{~g}$ of fat, it is difficult to accept that the $39 \%$ of the fat which is saturated is damaging to the cardiovascular system while the $61 \%$ of the fat which is unsaturated is protective. Keeping in mind that the total fat content of the steak will provide all but 3 of the 13 vitamins and 16 minerals that are a prerequisite for the maintenance of good health.

The verification for the saturated versus unsaturated fat theory is equivocal. Mozaffarian et al. [10] reported that postmenopausal women with relatively low total fat intake and a greater saturated fat intake were associated with less progression of coronary atherosclerosis. A recent meta-analysis [25] reviewing 347,747 people reported that there was no significant evidence for concluding that saturated fat is associated with an increased risk of CHD. The authors were of the opinion that more evidence should be gathered on the nutrients that would replace saturated fat. A prime candidate for this is sugar, which has tripled in consumption worldwide over the last 50 years and is implicated in the rapid rise in obesity. It is recommended that government intervention should be directed towards "added sugar". This is defined as any sweetener containing the molecule fructose that is added in food processing [26].

THoMP [24] refers to the COMA report [3], which recommends a ratio of polyunsaturated fats to saturated fat that is a P/S ratio of 0.45 . The egg is the only natural food with this ratio. Of the $10 \mathrm{~g}$ of fat per $100 \mathrm{~g}$ of egg, $37 \%$ is saturated, $46 \%$ is monounsaturated, and $17 \%$ is polyunsaturated [27]. Precise daily dietary recommendations are required to demonstrate how total fat, saturated fat and the P/S ratio can be met in parallel with micronutrient recommended daily allowances [RDAs].

\section{Swapping Dietary Fats-The Limitations and the Consequences}

The THoMP [24] report also recommended that the population goals should be to replace dietary saturated fats with small amounts of unsaturated fats. There are two ways in which this can be done; one has no impact on macronutrients but both methods will influence micronutrients. Sunflower oil, for example could replace olive oil and slightly reduce saturated fat while increasing polyunsaturated fat at the expense of monounsaturated fat
(Table 1). Very little saturated fat (4 g/100g) would be replaced by unsaturated fat. Vitamin E would increase from $14 \mathrm{mg}$ to $41 \mathrm{mg}$ while Vitamin $\mathrm{K}$ would fall from $60 \mathrm{mcg}$ to $5 \mathrm{mcg}$ [22,23]; these are the only two nutrients of small value in olive oil and sunflower oil. Oils generally, are deficient in vitamins and devoid of minerals.

Substituting foods other than oils, changes the relative amounts of saturated and unsaturated fat, calorie intake and the macro and micronutrients. Replacing $100 \mathrm{~g}$ of cheese with $100 \mathrm{~g}$ of olive oil will reduce saturated fats [SFAs] by $7 \mathrm{~g}$ and increase monounsaturated fats [MUFAs] eight fold (64 g) and PUFAs twelvefold. Calorie intake doubles while losing: $25 \mathrm{~g}$ of protein; $33 \%$ of vitamin A and B12 RDAs; $70 \%$ of the calcium and phosphorus RDAs and all zinc [28]. If whole milk is replaced with the same oil, all protein and minerals are lost, calories increase 15 fold and all three fats increase substantially SFAs seven fold, MUFAs 91 fold and PUFAs 55 fold [29].

Despite a vast amount of nutritional research over many decades we are still surrounded by confusion, rapidly changing advice and substantial mythology. Many of the contemporary eating habits are harmless but some, particularly in the young, can be a threat to good health. For instance a reduction in the consumption of dairy foods would seriously challenge the daily prerequisite levels of vitamin A and D and the key minerals calcium and phosphorus. There is evidence of a re-emergence of rickets and osteoporosis is rising [30]. The most recent Family Food survey has reported that the present UK diet is deficient in retinol and vitamin $\mathrm{D}$, providing less than one third of the UK Reference Nutrient Intake and barely a fifth of the recently revised American RDA for vitamin $\mathrm{D}$ [31].

The consumption of oily fish is now considered a must and caution is given over red meat. However if $100 \mathrm{~g}$ of pork is replaced with the same quantity of mackerel we double the intake of calories and saturated fat. The intake of MUFAs would triple and PUFAs increase almost seven fold $[32,33]$ with the protein remaining the same. Vitamins are gained and some minerals are gained and others lost.

\section{Conclusion}

Nutritional science despite many years of enquiry is in a state of equivocation, resulting in many false promises being given with reference to a diet which will keep the majority of the population fit lean and healthy. Despite the influence of epigenetics we remain hunter gatherers surrounded by a preponderance of food and automation. This presents us with many problems of adjustment but it is not as complicated to solve as some would have us believe, albeit with ulterior motives mostly financial. 
Table 1. Nutritional content of foods in article.

\begin{tabular}{|c|c|c|c|c|c|c|c|c|}
\hline (All per $100 \mathrm{~g})$ & Sirloin steak & Eggs & Sunflower oil & Olive oil & Cheese & Milk (whole) & Pork & Mackerel \\
\hline Water (g) & 71 & 76 & 0 & 0 & 37 & 88 & 75 & 64 \\
\hline Carbohydrate (g) & 0 & 1 & 0 & 0 & 1 & 5 & 0 & 0 \\
\hline Protein (g) & 21 & 13 & 0 & 0 & 25 & 3 & 21 & 19 \\
\hline Fat (g) & 5.4 & 8.3 & 100 & 100 & 31 & 2.9 & 3.8 & 12.1 \\
\hline SFA/MUFA/PUFA (g) & $2.1 / 3 / 0.3$ & 3.1/3.8/1.4 & $10 / 45 / 40$ & $14 / 73 / 11$ & 21/9/0.9 & $1.9 / 0.8 / 0.2$ & $1.5 / 1.8 / 0.5$ & $3.3 / 5.5 / 3.3$ \\
\hline Calories & 154 & 143 & 884 & 884 & 403 & 60 & 123 & 205 \\
\hline \multicolumn{9}{|l|}{ Vitamins USA RDA } \\
\hline A (3000 IU) & 0 & 487 & 0 & 0 & 1002 & 102 & 0 & 167 \\
\hline B1 (1.2 mg) & 0.1 & 0.1 & 0 & 0 & 0 & 0 & 0.5 & 0.2 \\
\hline B2 (1.3 mg) & 0.1 & 0.5 & 0 & 0 & 0.4 & 0.2 & 0.2 & 0.3 \\
\hline B3 (16 mg) & 7.2 & 0.1 & 0 & 0 & 0.1 & 0.1 & 8.6 & 9.1 \\
\hline B5 (5 mg) & 0.6 & 1.4 & 0 & 0 & 0.4 & 0.4 & 0.7 & 0.9 \\
\hline $\mathrm{B} 6$ (1.7 mg) & 0.6 & 0.1 & 0 & 0 & 0.1 & 0 & 0.7 & 0.4 \\
\hline Folic Acid (400 mcg) & 13 & 47 & 0 & 0 & 18 & 5 & 0 & 1 \\
\hline B12 (2.4 mcg) & 1.2 & 1.3 & 0 & 0 & 0.8 & 0.4 & 0.5 & 8.7 \\
\hline $\mathrm{C}(90 \mathrm{mg})$ & 0 & 0 & 0 & 0 & 0 & 0 & 0 & 0.4 \\
\hline D (600 IU) & 0 & 35 & 0 & 0 & 12 & 40 & 0 & 360 \\
\hline $\mathrm{E}(15 \mathrm{mg})$ & 0.3 & 1 & 41 & 14 & 0.3 & 0.1 & 0.1 & 1.5 \\
\hline K (120 mcg) & 1.2 & 0.3 & 5.4 & 60 & 2.8 & 0.2 & 0.1 & 5 \\
\hline \multicolumn{9}{|l|}{ Minerals (Macro) } \\
\hline Calcium (1000 - 1200 mg) & 27 & 53 & 0 & 0 & 721 & 113 & 5 & 12 \\
\hline Magnesium (420 mg) & 22 & 12 & 0 & 0 & 28 & 10 & 23 & 76 \\
\hline Phosphorus (700 mg) & 193 & 191 & 0 & 0 & 512 & 91 & 290 & 217 \\
\hline Potassium (4700 mg) & 323 & 134 & 0 & 0 & 98 & 143 & 479 & 314 \\
\hline Sodium (1500 mg) & 54 & 140 & 0 & 0 & 621 & 40 & 226 & 90 \\
\hline \multicolumn{9}{|l|}{ Minerals (Trace) } \\
\hline Copper (0.9 mg) & 0.1 & 0.1 & 0 & 0 & 0 & 0 & 0.1 & 0.1 \\
\hline Iron (18 mg) & 1.5 & 1.8 & 0 & 0 & 0.7 & 0 & 0.4 & 1.6 \\
\hline Selenium (55 mcg) & 24.1 & 31.7 & 0 & 0 & 14 & 3.7 & 39.6 & 44 \\
\hline Zinc (11 mg) & 3.9 & 1.1 & 0 & 0 & 3.1 & 0.4 & 1.4 & 0.6 \\
\hline
\end{tabular}

The highest value is highlighted for each macro and micro nutrient for prima facie observation.

There is a need to return to eating natural foods such as meat, eggs and dairy from grazing animals, fish, vegetables, nuts, seeds and local seasonal fruits. These were a feature of the British diet prior to a myriad of dietary advice based on questionable research, resulting in two thirds of the population becoming overweight/obese and sick within three decades.

\section{REFERENCES}

[1] A. Keys, "Coronary Heart Disease in Seven Countries: I. The Study Program and Objectives," Circulation, Vol. 41, No. 4S, 1970, pp. I-1-I-8. doi:10.1161/01.CIR.41.4S1.I-1

[2] J. P. Carter, "Eating in America: Dietary Goals for the United States: Report of the Select Committee on Nutrition and Human Needs US Senate," MIT Press, Cambridge, 1977.

[3] Committee on Medical Aspects of Food Policy (COMA),
"Diet and Cardiovascular Disease: Report of the Panel on Diet in Relation to Cardiovascular Disease,” 1984.

[4] National Advisory Committee on Nutritional Education (NACNE), “A Discussion Paper on Proposals for Nutritional Guidelines for Health Education in Britain,” 1983.

[5] National Institute of Clinical Excellence (NICE), "Prevention of Cardiovascular Disease at a Population Level, in Public Health Guidance 25,” 2010.

[6] W. S. Harris, et al., "Omega-6 Fatty Acids and Risk for Cardiovascular Disease: A Science Advisory from the American Heart Association Nutrition Subcommittee of the Council on Nutrition, Physical Activity, and Metabolism; Council on Cardiovascular Nursing; and Council on Epidemiology and Prevention," Circulation, Vol. 119, No. 6, 2009. pp. 902-907. doi:10.1161/CIRCULATIONAHA.108.191627

[7] A. Keys, "Coronary Heart Disease in Seven Countries: XVII. The Diet,” Circulation, Vol. 41, No. 4S, 1970, pp. I-162-I-183. 
[8] A. Menotti, et al., "Inter-Cohort Differences in Coronary Heart Disease Mortality in the 25-Year Follow-Up of the Seven Countries Study," European Journal of Epidemiology, Vol. 9, No. 5, 1993, pp. 527-536. doi:10.1007/BF00209531

[9] S. Tsimikas, et al., "LDL Isolated From Greek Subjects on a Typical Diet or From American Subjects on an OleateSupplemented Diet Induces Less Monocyte Chemotaxis and Adhesion When Exposed to Oxidative Stress," Arteriosclerosis, Thrombosis, and Vascular Biology, Vol. 19, No. 1, 1999, pp. 122-130. doi:10.1161/01.ATV.19.1.122

[10] D. Mozaffarian, E. B. Rimm and D. M. Herrington, "Dietary Fats, Carbohydrate, and Progression of Coronary Atherosclerosis in Postmenopausal Women," The American Journal of Clinical Nutrition, Vol. 80, No. 5, 2004, pp. 1175-1184.

[11] National Heart Lung and Blood Institute, "Third Report of the Expert Panel on Detection, Evaluation, and Treatment of High Blood Cholesterol in Adults (Adult Treatment Panel III)," 2002.

[12] G. A. Rose, W. B. Thomson and R. T. Williams, "Corn Oil in Treatment of Ischaemic Heart Disease,” BMJ, Vol. 1, No. 5449, 1965. pp. 1531-1533. doi:10.1136/bmj.1.5449.1531

[13] I. D. Frantz, et al., "Test of Effect of Lipid Lowering by Diet on Cardiovascular Risk. The Minnesota Coronary Survey," Arteriosclerosis, Thrombosis, and Vascular Biology, Vol. 9, No. 1, 1989, pp. 129-135. doi:10.1161/01.ATV.9.1.129

[14] "Low-Fat Diet in Myocardial Infarction: A Controlled Trial,” The Lancet, Vol. 2, No. 7411, 1965, pp. 501-504.

[15] “Controlled Trial of Soya-Bean Oil in Myocardial Infarction: Report of a Research Committee to the Medical Research Council,” The Lancet, Vol. 292, No. 7570, 1968, pp. 693-700. doi:10.1016/S0140-6736(68)90746-0

[16] S. Dayton, et al., "Controlled Trial of a Diet High in Unsaturated Fat for Prevention of Atherosclerotic Complications,” The Lancet, Vol. 292, No. 7577, 1968, pp. 10601062. doi:10.1016/S0140-6736(68)91531-6

[17] P. Leren, “The Oslo Diet-Heart Study,” Circulation, Vol. 42, No. 5, 1970, pp. 935-942. doi:10.1161/01.CIR.42.5.935

[18] J. M. Woodhill, et al., "Low Fat, Low Cholesterol Diet in Secondary Prevention of Coronary Heart Disease," Advances in Experimental Medicine and Biology, Vol. 109, 1978, pp. 317-330. doi:10.1007/978-1-4684-0967-3 18

[19] G. F. Watts, et al., "Effects on Coronary Artery Disease of Lipid-Lowering Diet, or Diet Plus Cholestyramine, in the St Thomas' Atherosclerosis Regression Study (STARS)," The Lancet, Vol. 339, No. 8793, 1992, pp. 563-569. doi:10.1016/0140-6736(92)90863-X

[20] United States Department of Agriculture, H.N.R.C., "Composition of Foods, Raw, Processed, Prepared, USDA National Nutrient Database for Standard Reference, Release 21,” 2008.

[21] United States Department of Agriculture, H.N.R.C., "Beef, Bottom Sirloin, Tri-Tip, Separable Lean Only, Trimmed to 0" Fat, Choice, Raw [URMIS \#2244],” 2008.

[22] United States Department of Agriculture, H.N.R.C., "Oil, Vegetable, Sunflower, Linoleic (Less than 60\%),” 2008.

[23] United States Department of Agriculture, H.N.R.C., “Oil, Olive, Salad or Cooking,” 2008.

[24] National Institute of Clinical Excellence (NICE), "Prevention of Cardiovascular Disease at a Population Level: Evidence on Interventions to Address Dietary Fats, in NICE CVD Guidance Expert Testimony Paper 10,” 2009.

[25] P. W. Siri-Tarino, et al., "Meta-Analysis of Prospective Cohort Studies Evaluating the Association of Saturated Fat with Cardiovascular Disease,” The American Journal of Clinical Nutrition, Vol. 91, No. 3, 2010, pp. 535-546. doi:10.3945/ajcn.2009.27725

[26] R. H. Lustig, L. A. Schmidt and C. D. Brindis, "Public Health: The Toxic Truth about Sugar,” Nature, Vol. 482, No. 7383, 2012, pp. 27-29. doi:10.1038/482027a

[27] United States Department of Agriculture, H.N.R.C., "Egg, Whole, Raw, Fresh," 2008.

[28] United States Department of Agriculture, H.N.R.C., "Cheese, Cheddar," 2008

[29] United States Department of Agriculture, H.N.R.C., "Milk, Whole 3.25\% Milk Fat,” 2008.

[30] S. H. Pearce and T. D. Cheetham, "Diagnosis and Management of Vitamin D Deficiency,” BMJ, Vol. 340, 2010, p. b5664. doi:10.1136/bmj.b5664

[31] DEFRA (The Department for Environment, Food and Rural Affairs), "A National Statistics Publication, the Family Food Survey,” The Department for Environment, Food and Rural Affairs, London, 2010.

[32] United States Department of Agriculture, H.N.R.C., "Pork, Fresh, Enhanced, Loin, Top Loin (Chops), Boneless, Separable Lean and Fat, Raw,” 2008.

[33] United States Department of Agriculture, H.N.R.C., "Fish, Mackerel, Atlantic, Raw,” 2008. 